\title{
BASF Constructs New Research Building
}

B ASF is constructing a new laboratory building close to Gate 7 in the south of the Ludwigshafen site on an area covering 4300 square metres. It will offer material physicists and additive, formulation and polymer researchers ideal conditions for even closer cooperation. The investment amounts to approximately 50 million euros.

Dr. Andreas Kreimeyer, Member of the Board of Executive Directors, Re- search Executive Director, said: "Innovations based on efficient and effective research and development are an important cornerstone of our strategy in the future and will be the guarantor of organic growth. With the new research building we will keep our site in Ludwigshafen competitive on an international level and strengthen our global research network."

In constructing the new laboratory facilities, which will be ready for occu-

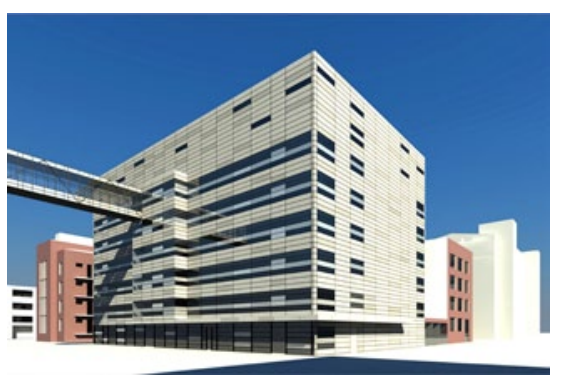

pancy in the second half of 2015, BASF attaches particular value to the use of energy-efficient solutions.

\section{The Fraunhofer CBP: Industrial Chemicals from Renewable Raw Materials}

The new Fraunhofer Centre for Chemical-Biotechnological Processes CBP took just under two years to build and was formally opened recently in the presence of German Chancellor Angela Merkel. The new institute is intended to bridge the gap in the use of renewable raw materials between the laboratory and industrial production.

A dhesives, plastics and coatings, together with many important intermediate products in the chemical industry, are still primarily manufactured from mineral oil. However, chemical companies throughout the world are working on replacing oil with renewable raw materials. Some of these new processes, which do not make use of food or feed crops, already function well on a laboratory scale. But the use of renewable raw materials in mass production processes requires a significant financial and technological investment even for large companies. Small and medium-sized firms have often failed to bring their products into use in indus-

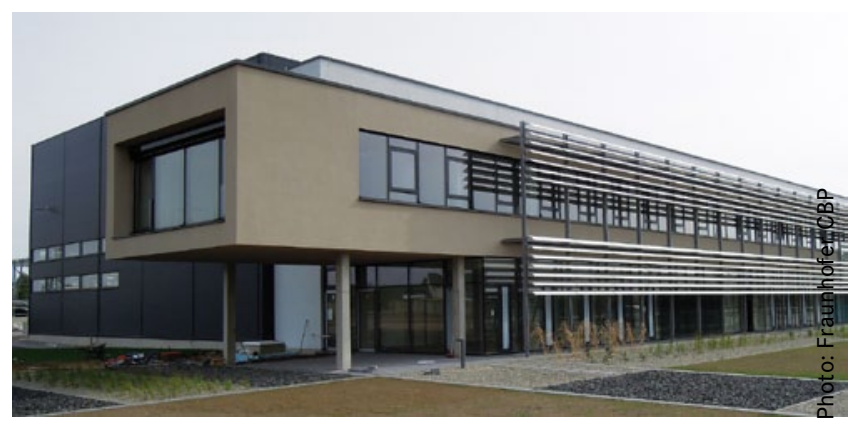

The newly completed Fraunhofer Centre for ChemicalBiotechnological Processes CBP. try, despite their successes in the laboratory. The new Fraunhofer Centre for Chemical-Biotechnological Processes CBP, which was formally opened on 2 October 2012 after a construction period of less than two years, aims to change this situation.

"The new Fraunhofer Centre for Chemical-Biotechnological Processes CBP bridges the gap in the use of renewable raw materials between the laboratory and industrial production," explained Professor Reimund Neugebauer, who took up his new role as president of the Fraunhofer-Gesellschaft on $1 \mathrm{OC}^{-}$ tober 2012. "Manufacturing companies are still largely dependent on fossil raw materials. By using renewable raw materials we can reduce this dependency and cut $\mathrm{CO}_{2}$ emissions at the same time. The Fraunhofer CBP will also enable processes to be introduced that are signifi- cantly more efficient in their use of raw materials and energy than existing petrochemical-based methods. This will move us much closer towards establishing a sustainable chemical industry."

The Fraunhofer CBP will be staffed jointly by scientists from the Fraunhofer Institutes for Interfacial Engineering and Biotechnology IGB (Stuttgart) and for Chemical Technology ICT (Pfinztal). Following a two-year development phase, a total of 19 people are already working at the CBP.

Five different process plants are available, based on the model of a biorefinery, which can be operated separately or easily combined, as required. This highly flexible concept offers new opportunities for expanding the use of biological raw materials and introducing them into industrial-scale production. This will make it possible to combine existing processes in the chemical industry with new and innovative procedures and to produce raw materials containing oil, lignin, cellulose, starch or sugar that can be used as the basis for manufacturing products. 